\title{
Proceedings
}

\section{Improving Numerical Estimation of Cyclist Drag Area in Static Conditions Using Unsteady RANS +}

\author{
Ardalan Javadi ${ }^{1}$, Andrew J. M. Buckrell ${ }^{1,2}$ and Sean D. Peterson ${ }^{1, *}$ \\ 1 Mechanical and Mechatronics Engineering Department, University of Waterloo, 200 University Avenue \\ West, Waterloo, ON N2L 3G1, Canada; ardalan.javadi@uwaterloo.ca (A.J.); \\ andrew.buckrell@4iiii.com (A.J.M.B.) \\ 2 4iiii's Innovations, Inc., 1412 Avenue East, Cochrane, AB T4C 2B9, Canada \\ * Correspondence: peterson@uwaterloo.ca; Tel.: +1-519-888-4567 (ext. 38722) \\ + Presented at the 13th conference of the International Sports Engineering Association, Online, \\ 22-26 June 2020.
}

Published: 15 June 2020

\begin{abstract}
Herein, we compare the drag area estimated using unsteady Reynolds-averaged NavierStokes (URANS), using the $\gamma-R e \Theta$ transitional shear stress transport (SST) $k-\omega$ (SSTLM) turbulence model with published experimental measurements of a static full-scale cyclist mannequin in an open test section wind tunnel, with the left leg fully extended. The turbulence model employs a local empirical correlation based upon a classical Blasius boundary layer behavior to predict flow transition. For a given mesh density, we aim to improve drag area estimation by modifying the empirical correlation coefficient to better capture actual boundary layer transition location around the arms and legs, to facilitate computationally economical cyclist simulations. Large Eddy Simulation (LES), in conjunction with experimental wake data in the vicinity of the arms and legs, is used to assess boundary layer shape factors, which are related to the empirical coefficient. Overall, the drag area predicted by LES is within 3.7\% of the measured results, while the original SSTLM is within $7.8 \%$. By tuning the correlation coefficient, the drag area error is improved to $6.0 \%$ at no additional computational cost. The tuning was relatively coarse, and was only considered for the appendages. In other regions, the original SSTLM coefficient seems to perform better, suggesting that local coefficient selection may lead to further improvements in results over the currently employed global value.
\end{abstract}

Keywords: transitional turbulence modelling; wake flow; drag force; cycling

\section{Introduction}

Highly resolved computational fluid dynamics (CFD) has gained popularity as a tool for aerodynamic assessment in sport over the past decade or so, with considerable effort focused on cycling [1-3]. CFD affords practitioners insights into the detailed flow features involved in producing observed bulk effects, such as drag. Simulating flow around a full cyclist is challenging, however, owing to issues such as geometric complexity, flow separations and critical flow regions susceptible to laminar-to-turbulent transition. Furthermore, a wide range of small scale surface roughness characteristics and non-linear textile response compound the issues numerically. To this end, considerable effort has been devoted to assessing the performance of various turbulence modeling strategies for cyclist aerodynamics [3-5]. Defraeye et al. [5] studied various cyclist postures using Reynolds Averaged Navier-Stokes (RANS) with a low Reynolds number $k-\varepsilon$ turbulence model and Large Eddy Simulation (LES), and found that RANS under-predicted drag area $\left(C_{d} A\right)$ by $11 \%$, whereas LES under-predicted by $7 \%$ in comparison with wind tunnel experiments. Fintelman et al. [6] studied a cyclist in crosswinds using RANS, Detached Eddy Simulation (DES) and LES, and found 
that a SST k- $\omega$ turbulence model can capture the development of large flow separation around the bicycle with increasing yaw angles. Blocken et al. [Error! Reference source not found.], as part of a broader effort to study cycling pelotons, modeled flow around a single cyclist using the $\gamma-R e \Theta$ transitional SST $k-\omega$ (SSTLM) turbulence model proposed for transitional flows [7]. By ensuring small wall-adjacent cell sizes in the cyclist mesh, their estimated drag area was within $0.8 \%$ of analogous wind tunnel measurements. This exceptional result required a very highly resolved grid, with approximately 40 elements in the boundary layer around each cyclist, and consequently was extremely computationally expensive; the simulations employed about 14,000 CPUs in a Cray XC-40 supercomputer to generate the results.

In this paper, we aim to improve the drag area estimate around a single cyclist using unsteady RANS (URANS) by tuning the SSTLM turbulence model to better predict flow transition and separation around the rider appendages with relatively coarse grid density and modest computational expense. LES is used to evaluate boundary layer characteristics in these regions, which drives the local behavior of the turbulence model.

\section{Materials and Methods}

The cyclist mannequin and bike model employed in the experimental study by Terra et al. [Error! Reference source not found.] were numerically replicated herein, and the flow around the model was evaluated using both URANS and LES. The Reynolds number of the experiments and simulations based on rider length, $L=1.7 \mathrm{~m}$, is $5 \times 10^{5}$, with a free stream velocity of $U_{\infty}=12.95 \mathrm{~m} / \mathrm{s}$. Error! Reference source not found. shows the cyclist model, along with mesh details on the lower wall and in the vicinity of the rider for a representative case. The domain dimensions are $L_{x} \times L_{y} \times L_{z}$ $=7.5 \mathrm{~L} \times 3.5 \mathrm{~L} \times 3.5 \mathrm{~L}$, where $x$ is the streamwise direction and $y$ is the wall-normal direction. The appropriate wall spacing for the very first cell close the body for the considered Reynolds number is $20 \mu \mathrm{m}$ to achieve $y^{+} \sim 1$. The wall spacing should be reduced to one tenth of this value in stagnation zones, such as the frontal area of the helmet, arms and legs to achieve $y^{+} \sim 1$. The wall spacing herein is ave $^{+} \sim 4$, placing the first few cells in the viscous sublayer. The boundary layer contains 18 cells, and the total number of elements for URANS and LES are 43 million and 56 million, respectively. The inlet flow is of uniform velocity, the rear boundary is stress free, and the top and side walls are free slip. To faithfully replicate the experimental study, the ground plane and wheels are stationary.

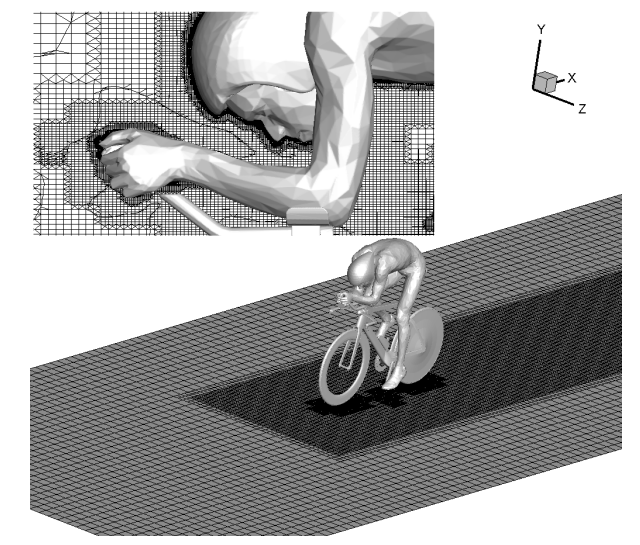

Figure 1. Isometric view of the cyclist model and mesh density on the ground plane. The inset shows a representative example of the mesh details in the immediate vicinity of the rider's arms and head.

Two turbulence modelling approaches were used herein; the dynamic $k$-equation model was used in LES, and the SSTLM turbulence model was used in URANS. The latter model uses a local empirical correlation-based approach to estimate transition location. The empirical coefficient, $C=$ 2.193, relating momentum thickness Reynolds number, $\operatorname{Re} \theta$, and vorticity Reynolds number, $\operatorname{Re} \Omega$, holds for boundary layer shape factors, $H$, between 2.3 and 2.9. The Reynolds numbers of the upper arm and thigh, based on mean diameter, are $1.3 \times 10^{5}$ and $2.85 \times 10^{5}$, respectively, suggesting that flow 
around these regions is susceptible to transition, particularly since the incoming turbulence intensity may be elevated due to flow disruptions upstream by other rider and bike elements.

Computations were performed using OpenFOAM-1906, a finite volume open source CFD code. Central differencing was used for the diffusion terms, whereas a limited linear total variation diminishing scheme with a conformance coefficient was used for convective terms. Two approaches were employed to treat the flow close to the wall; two-layer wall modeling (TLM) and Spalding's law. The simulations were performed using $2.4 \mathrm{GHz}$ Intel cores with 200 GB RAM. Results were averaged over approximately $2.5 \mathrm{~s}$ of physical time.

\section{Results and Discussion}

Table 1 compares the drag area, $C_{d} A$, of the experiment with the predicted values from the numerical simulations using both wall treatment approaches. The experimental value was reduced by $1 \%$, to account for the contribution to the drag of the fixed bars between the force plate and the wheel hub to support the mannequin and bike in the experiments, which is not included in the numerical simulations. Furthermore, the rear wheel gear, the chain and the front wheel spokes were simplified in the numerical simulations, which additionally reduced the simulated drag. The uncertainty in the experimental results was also estimated from details reported in Terra et al. [Error! Reference source not found.] and included in Table 1. The LES results under-predicted $C_{d} A$ by $3.7 \%$ using TLM, which was the lowest of the models considered; URANS with SSTLM under-predicted $C_{d} A$ by $7.8 \%$. For LES, TLM produces better results in comparison with Spalding's law, whereas the trend is reversed for URANS, with Spalding's law yielding slightly better results than TLM. Table 1 also highlights the relative expense of LES versus URANS with SSTLM, with the latter affording roughly $10 \%$ savings in terms of CPU hours.

Table 1. Estimated drag area and computational cost.

\begin{tabular}{cccccc}
\hline \multirow{2}{*}{ Model } & \multicolumn{2}{c}{$\left.\boldsymbol{C}_{d} \boldsymbol{A} \mathbf{( m}^{2}\right)$} & \multirow{2}{*}{ Error } & \multirow{2}{*}{ CPU.hr } & \multirow{2}{*}{ Time Step (s) } \\
\cline { 2 - 4 } & TLM & Spalding's Law & & \\
\hline Experiment & 0.217 & - & $1.5 \%{ }^{\mathrm{a}}$ & - & - \\
LES & 0.209 & 0.207 & $3.7 \%^{\mathrm{b}}$ & 17,200 & $5 \times 10^{-5}$ \\
SSTLM & 0.198 & 0.200 & $7.8 \% \mathrm{~b}$ & 15,500 & $1 \times 10^{-4}$ \\
Tuned SSTLM & - & 0.204 & $6.0 \%$ & 15,500 & - \\
\hline
\end{tabular}

${ }^{\mathrm{a}}$ uncertainty of experimental measurements; ${ }^{\mathrm{b}}$ best from TLM or Spalding's law; ${ }^{\mathrm{c}} \mathrm{C}=1.6$.

Along the vertical appendages of the model, detailed comparison of the results shows that LES predicts flow transition further upstream than does URANS with SSTLM. The LES results indicate that the boundary layer shape factor decreases from $H=2.1$ in the vicinity of stagnation to a minimum of around 1.2, and then increases to approximately 4 at separation. As previously stated, the correlation coefficient $C=2.193$ relating $\operatorname{Re} \theta$ and $\operatorname{Re} \Omega$ is valid for $2.3<H<2.9$ [7], but is too high for shape factors out of this range, leading to transition being predicted too far downstream. In an effort to improve the estimate of the transition location in the SSTLM model, three correlation constants are considered, $C=1.8,1.6$ and 1.4, and compared with the original SSTLM results.

Error! Reference source not found. compares the results of the original and the tuned SSTLM model with experimental velocity measurements in the wake of upper arm and the extended leg. The measurement for the arm is at $y=1.1 \mathrm{~m}$, and for the thigh is at $y=0.95 \mathrm{~m}$. Both are extracted $x / d=0.8$ downstream of the cross-section center, where $d$ is the width of the cross-section. The root mean square errors between the predictions and experimental data are $1.1 \%, 0.5 \%, 1.1 \%$ and $0.9 \%$ for $\mathrm{C}=$ 1.4, 1.6, 1.8 and 2.193 (original), respectively, for the arm (Figure 2a), and 1.9\%, 1.3\%, 1.4\% and 1.5\% for the thigh (Figure 2b). A value of $C=1.6$ shows the best agreement for both appendages, with the most significant improvement for the flow around the upper arm. For the remainder of the manuscript, $C=1.6$ will be employed as the 'Tuned SSTLM' model. Table 1 shows that, with tuning, the estimate of $C_{d} A$ improves from $7.8 \%$ error to $6.0 \%$. 


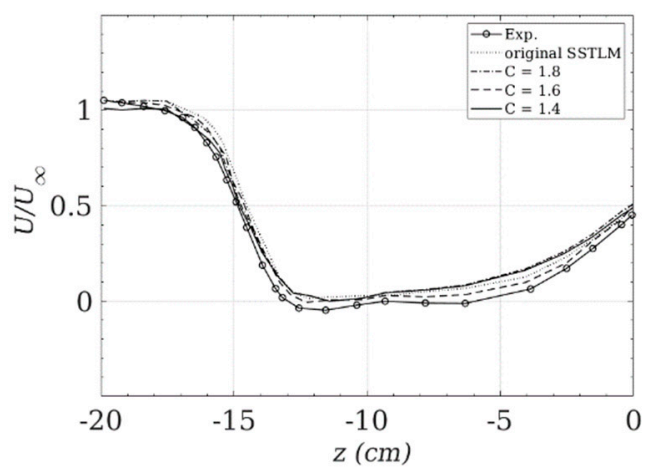

(a)

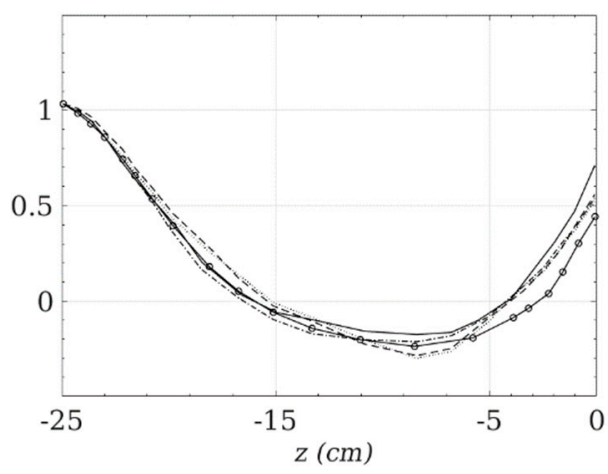

(b)

Figure 2. Tuned SSTLM model, $\operatorname{Re} \theta=\operatorname{Re} \Omega / C$, compared with experimental measurements in the wake of (a) the upper arm and (b) the thigh of the extended leg. The measurement for the arm is at $y=1.1$ $\mathrm{m}$, and for the thigh is at $y=0.95 \mathrm{~m}$, and $x / d=0.8$ downstream of the cross-section center. $d$ is the width of the cross-section.

Error! Reference source not found. compares the contour of $0.90 U_{\infty}$ for the different turbulence modeling approaches, using TLM in a yz-plane $0.8 \mathrm{~m}$ behind the rear wheel, with the experimental data of Terra et al. [8]. In general, the wake width is reasonably captured for both the LES and URANS methods, though URANS predicts the separation over the hip further downstream than does LES. The tuned SSTLM improves the prediction of the separation on the raised leg side of the hip (positive $z$ ); see Error! Reference source not found.b. Stagnation regions with their strong pressure gradients, three-dimensional flow transition after stagnation, and separation over the hip are challenging to predict. LES predicts the torso wake well $(z>100)$, while it under-predicts the coherent structures generated from the feet ( $z \sim 40$ and 60 for the left and right foot, respectively).

Overall, URANS with SSTLM predicts the large scale wake structures well; see Error! Reference source not found.a. The intermittency equation in SSTLM needs very fine resolution close to the wall, with a zero normal flux boundary condition. It is only sensitive to streamwise velocity over the surface. The tuned model increases the intermittency, which increases the production of turbulent kinetic energy further upstream. The SST $k-\omega$ model limits the eddy viscosity to improve the performance of the model for adverse pressure gradients and in the wake regions. Nevertheless, SSTLM, which was developed for attached transitional flows, predicts a slightly more diffusive wake compared with the LES results; see Error! Reference source not found.a.

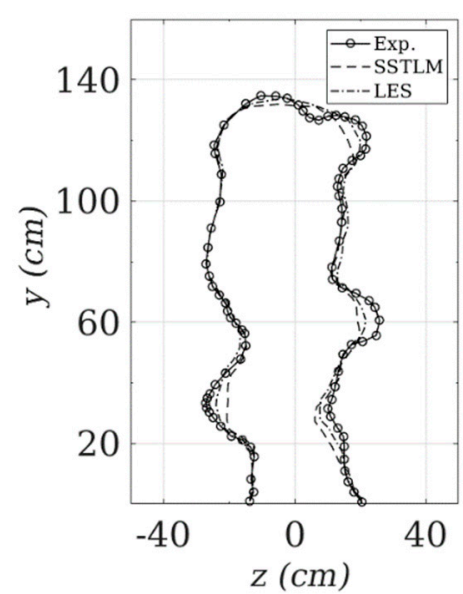

(a)

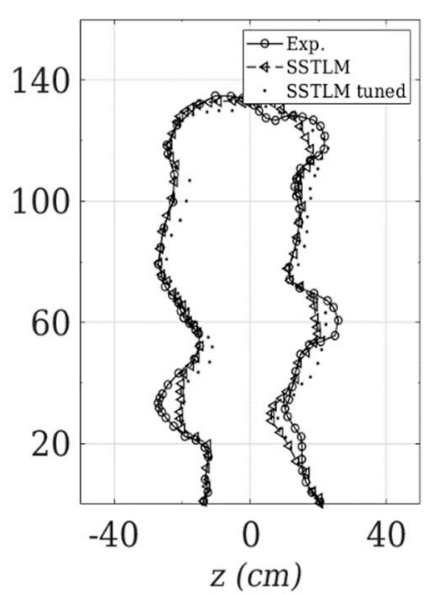

(b)

Figure 3. Contour of $0.9 U_{\infty}$ for different meshes and turbulence modeling approaches in a $y z$-plane at $0.8 \mathrm{~m}$ behind the rear wheel, compared with the experimental data from Terra et al. [8]: (a) LES vs. SSTLM; (b) SSTLM vs. Tuned SSTLM. 
Error! Reference source not found. shows the pressure coefficient, $C_{p}=\left(p-p_{\infty}\right) / 0.5 \rho U_{\infty^{2}}$, in the same plane as Error! Reference source not found.Error! Reference source not found. for the experimental and simulation data. Error! Reference source not found.a shows that there is a high pressure region in $100<z<140$, which is downstream of the rider's torso. This pressure rise corresponds to the Bernoulli effect associated with the decrease in mean velocity in the wake of the cyclist. It also shows three low pressure regions in $80<z<100$. These regions correspond with three main coherent structures in the wake of the cyclist. The strongest one, which is on the $z$-axis, is generated from the hip; the left one (negative $z$ ) is generated from the outer part of extended leg; and the right one (positive $z$ ) is generated from the outer part of raised leg. The low pressure region on the extended leg side is spatially larger than that of the raised leg. There are three other low pressure regions in $z<60$, two of which are due to the coherent structures generated by the feet, and the remaining one is from the knee of the extended leg. The pressure coefficients from LES and SSTLM correspond well with the experimental measurements, with LES showing the best agreement; see Error! Reference source not found.b. The low pressure regions predicted by SSTLM are smaller than those of the experimental measurement (see Error! Reference source not found.c, indicating that SSTLM predicts smaller coherent structures than LES.

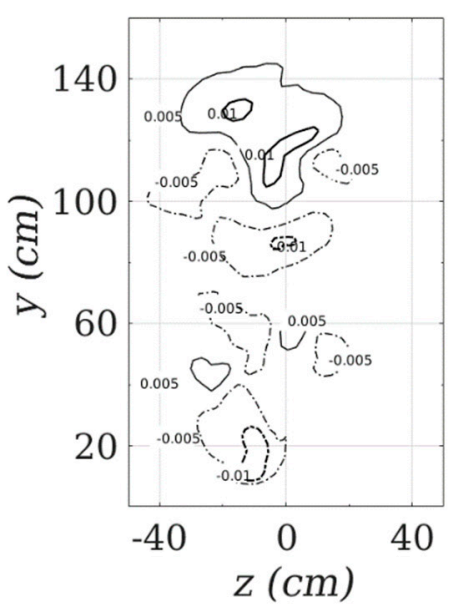

(a)

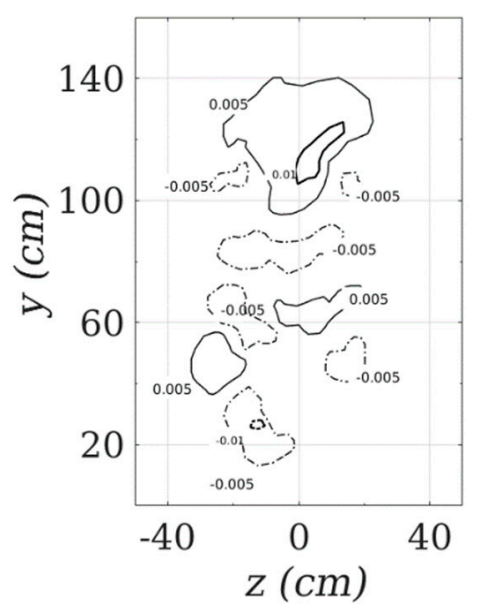

(b)

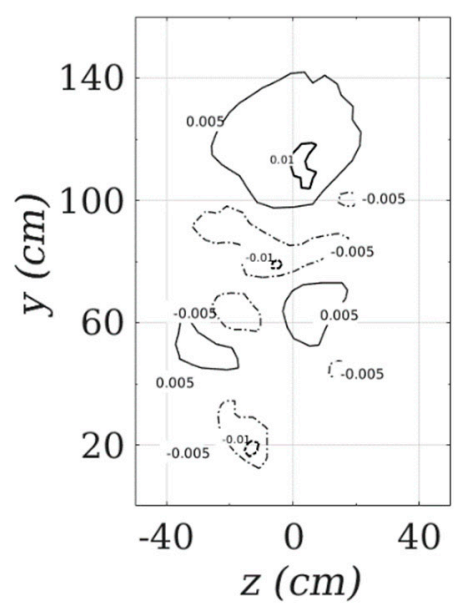

(c)

Figure 4. Contour of the pressure coefficient, $C_{p}=\left(p-p_{\infty}\right) / 0.5 \rho U_{\infty^{2}}$, at $0.8 \mathrm{~m}$ downstream of the rear wheel: (a) experimental measurements; (b) LES; (c) Tuned SSTLM.

\section{Conclusions}

The flow around a cyclist was studied using two methods, URANS and LES, and two near wall treatments: two-layer wall modeling and Spalding's law. The SSTLM turbulence model was used for the URANS simulations, as the flow was expected to be transitional around portions of the body. LES better predicts the drag area when using two-layer wall modelling, while Spalding's law performs better for URANS. The LES drag area estimation is within 3.6\% of the measured value, albeit at higher computational expense than URANS. URANS with SSTLM under-predicts the drag area by $7.8 \%$. Examining the transition location around the appendages predicted by LES and SSTLM revealed that LES predicts transition further upstream. To rectify this, the SSTLM model was tuned, which resulted in an improvement in drag area estimation of $23 \%$, in comparison with the original model, at no additional computational expense. Further revision of the SSTLM turbulence model, perhaps with the local values of the correlation coefficient, may provide additional improvements.

Funding: This research was funded by Own The Podium and MITACs under grant number IT13479.

Acknowledgments: The authors would like to thank W. Terra, A. Sciacchitano and Y.H. Shah at TU Delft for providing the geometry. This work was supported by MITACs and Own the Podium. 
Conflicts of Interest: The authors declare no conflict of interest. The funding sponsors had no role in the design of the study; in the collection, analyses, or interpretation of data; in the writing of the manuscript, or in the decision to publish the results.

\section{References}

1. Godo, M.; Corson, D.; Legensky, S. A practical analysis of unsteady flow around a bicycle wheel, fork and partial frame using CFD. In Proceedings of the 49th AIAA Aerospace Sciences Meeting including the New Horizons Forum and Aerospace Exposition, Orlando, FL, USA, 4-7 January 2011; p. 1237, doi:10.2514/6.2011-1237

2. Mannion, P.; Toparlar, Y.; Blocken, B.; Clifford, E.; Andrianne, T.; Hajdukiewicz, M. Analysis of crosswind aerodynamics for competitive hand-cycling. J. Wind. Eng. Ind. Aerodyn. 2018, 180, 182-190, doi:10.1016/j.jweia.2018.08.002.

3. Blocken, B.; Van Druenen, T.; Toparlar, Y.; Malizia, F.; Mannion, P.; Andrianne, T.; Marchal, T.; Maas, G.J.; Diepens, J. Aerodynamic drag in cycling pelotons: New insights by CFD simulation and wind tunnel testing. J. Wind. Eng. Ind. Aerodyn. 2018, 179, 319-337, doi:10.1016/j.jweia.2018.06.011.

4. Beaumont, F.; Taiar, R.; Polidori, G.; Trenchard, H.; Grappe, F. Aerodynamic study of time-trial helmets in cycling racing using CFD analysis. J. Biomech. 2018, 67, 1-8, doi:10.1016/j.jbiomech.2017.10.042.

5. Defraeye, T.; Blocken, B.; Koninckx, E.; Hespel, P.; Carmeliet, J. Aerodynamic study of different cyclist positions: CFD analysis and full-scale wind-tunnel tests. J. Biomech. 2010, 43, 1262-1268, doi:10.1016/j.jbiomech.2010.01.025.

6. Fintelman, D.; Hemida, H.; Sterling, M.; Li, F.-X. CFD simulations of the flow around a cyclist subjected to crosswinds. J. Wind. Eng. Ind. Aerodyn. 2015, 144, 31-41.

7. Langtry, R.B.; Menter, F.R. Correlation-Based Transition Modeling for Unstructured Parallelized Computational Fluid Dynamics Codes. AIAA J. 2009, 47, 2894-2906, doi:10.2514/1.42362.

8. Terra, W.; Sciacchitano, A.; Shah, Y.H. Aerodynamic drag determination of a full-scale cyclist mannequin from large-scale PTV measurements. Exp. Fluids 2019, 60, 29, doi:10.1007/s00348-019-2677-6.

(C) 2020 by the authors. Licensee MDPI, Basel, Switzerland. This article is an open access article distributed under the terms and conditions of the Creative Commons Attribution (CC BY) license (http://creativecommons.org/licenses/by/4.0/). 